\title{
LETTER TO THE EDITOR \\ Estimating the autonomic function from heart rate variability in mechanically ventilated patients after spinal cord injury
}

\author{
Spinal Cord (2015) 53, 838; doi:10.1038/sc.2015.97; published online 2 June 2015
}

We read with interest the paper by Malmqvist et al. ${ }^{1}$ in which acute changes in the cardiac autonomic tone after a spinal cord injury (SCI) were assessed by heart rate variability (HRV) analysis. The authors employed a commercial HRV software to quantify spectral powers in predefined frequency bands and, in this regard, we noted a possible methodological error due to the application of the default settings of the commercial software in the specific clinical condition of ventilated SCI patients.

In fact, the authors quantified the high-frequency power (HFP, 'normally defined as a marker of vagal modulation determined by the frequency of breathing', page 55) by integrating the power spectrum between 0.15 and $0.40 \mathrm{~Hz}$; and the low-frequency power (LFP, 'a combination of sympathetic and parasympathetic activity', page 55) by integrating the power spectrum over a contiguous band at lower frequencies $(0.04-0.15 \mathrm{~Hz})$. These bands were indicated by the Task Force of the European Society of Cardiology and the North American Society of Pacing and Electrophysiology on its guideline on $\mathrm{HRV},{ }^{2}$ and appear as the default frequency bands for spectral analysis in most HRV software packages. We remind that this Task Force identified the $0.15-0.40-\mathrm{Hz}$ frequency range as the spectral band that includes the respiratory rate in most of spontaneously breathing people. However, $34 \%$ of SCI patients were not breathing spontaneously, being under ventilator treatment (see Table 1 in Malmqvist et al. ${ }^{1}$ ). It should be considered that respiratory rates between 8 and 12 breaths $\min ^{-1}$ are recommended for mechanically ventilated patients not requiring hyperventilation. ${ }^{3}$ Therefore by setting the lower limit of the HFP band at $0.15 \mathrm{~Hz}$ (that corresponds to 9 breaths $\min ^{-1}$ only), the power of the respiratory oscillation will be associated to the lowfrequency band, and not to the high-frequency one, in the fraction of patients ventilated mechanically at the lower breathing rates.

The waterfall spectrogram of Figure 1 suggests that ventilator frequencies below or at the limit of the HFP band may have been used in the study by Malmqvist et al., ${ }^{1}$ because the first spectrum of the series shows a respiratory peak exactly at $0.15 \mathrm{~Hz}$, that is, the frequency separating LFP from HFP. Therefore, only half of the power of this respiratory peak was quantified as HFP, whereas the remaining power contributed to the LFP, in this way underestimating the vagal tone and overestimating the sympatho/vagal balance.

The authors employed a Hanning tapering window to reduce spectral leakage (page 55). Unfortunately, this window is of little use when the spectral peak is very close to one end of the frequency band.
In fact, the Hanning window decreases power leakage from the side lobes at the cost of an increased width of the main lobe, and it is the main lobe that may leak power from HFP to LFP when it overlaps the border between the bands. For the Hanning window, the width of the main lobe is $4 / T \mathrm{~Hz}$, with $T$ the window length in seconds. ${ }^{4}$ Assuming that R-R intervals have been sampled evenly at $5 \mathrm{~Hz}$ (a detail not provided in methods by the authors), the duration of the 2048-point Hanning window is $T=409.6 \mathrm{~s}$ and the width of the main lobe is about $0.01 \mathrm{~Hz}$. This means that significant errors may occur in the estimation of HFP and LFP when the rate of the mechanical ventilator is lower than 9.3 breaths $\min ^{-1}$.

Therefore we conclude that when mechanical ventilators are used, as in the present study, the default settings of commercial HRV analysis software packages can be safely used if the breathing rate is set $\geqslant 10$ breaths $\min ^{-1}$. For lower breathing rates, default settings may provide erroneous estimates. In this case, we suggest to center the HRV respiratory band at the frequency of the mechanical ventilator and to set its bandwidth to $4 / \mathrm{THz}$; at the same time, the lowfrequency band should not extend over the respiratory band as defined above.

\section{CONFLICT OF INTEREST}

The authors declare no conflict of interest.

\author{
P Castiglioni ${ }^{1}$ and G Merati ${ }^{2}$ \\ ${ }^{1}$ IRCCS Fondazione Don Carlo Gnocchi, Milan, Italy and \\ ${ }^{2}$ Department of Biomedical Sciences for Health, \\ University of Milan, Milan, Italy \\ E-mail: pcastiglioni@dongnocchi.it
}

1 Malmqvist L, Biering-Sørensen T, Bartholdy K, Krassioukov A, Welling KL, Svendsen JH et al. Assessment of autonomic function after acute spinal cord injury using heart rate variability analyses. Spinal Cord 2015; 53: 54-58.

2 Task Force of the European Society of Cardiology and the North American Society of Pacing and Electrophysiology. Heart rate variability. Standards of measurement, physiological interpretation, and clinical use. Eur Heart J 1996; 17: 354-381.

3 Amitai A. Ventilator management. Medscape Reference 2011. http://emedicine. medscape.com/article/810126-overview\#aw2aab6b7.

4 Bendat JS, Piersol AG. Random Data. Analysis and Measurement Procedures. John Wiley and Sons, New York, USA, 1986. 